Recepción: 15/ 05 / 2018

Aceptación: 19/ 08 / 2018

Ciencias técnicas y aplicadas

Publicación: 05/ 10 / 2018

Artículo de investigación

\title{
Caracterización y determinantes del rendimiento académico de estudiantes de ciencias Agropecuarias, Universidad de Cuenca
}
Characterization and determinants of the academic performance of students of agricultural sciences, University of Cuenca

\section{Caracterização e determinantes do desempenho acadêmico de estudantes de ciências agrárias, Universidade de Cuenca}

\author{
Cornelio A. Rosales-Jaramillo ${ }^{\text {I }}$ \\ cornelio.rosales@ucuenca.edu.ec \\ Yolanda M. Aguilar-Valladares II \\ yolanda.aguilarv@ucuenca.edu.ec \\ Ana L. Vásquez-Zamora ${ }^{\text {III }}$ \\ alucia.vazquezz@ucuenca.edu.ec \\ Clelia K. Guzmán-Espinoza IV \\ kathrine.guzman@ucuenca.edu.ec
}

Correspondencia: cornelio.rosales@ucuenca.edu.ec

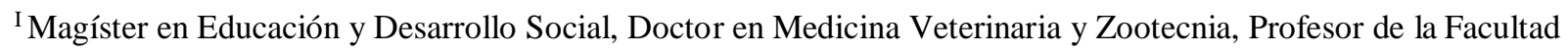
de Ciencias Agropecuarias, Universidad de Cuenca, Cuenca, Ecuador.

II Magíster en Educación, Doctora en Bioquímica y Farmacia, Profesora de la Facultad de Ciencias Agropecuarias, Universidad de Cuenca, Cuenca, Ecuador.

III Magíster en Educación, Arquitecta, Profesora de la Facultad de Ciencias Agropecuarias, Universidad de Cuenca, Cuenca, Ecuador.

${ }^{\text {IV }}$ Magíster en Ciencias de Gerencia y Administración Empresarial Agropecuaria, Ingeniera Agrónoma, Profesora de la Facultad de Ciencias Agropecuarias, Universidad de Cuenca, Cuenca, Ecuador.
} 


\section{Resumen}

La presente investigación descriptiva correlacional y transversal, se realizó en la Facultad de Ciencias Agropecuarias de la Universidad de Cuenca, Carreras de Medicina Veterinaria y Zootecnia e Ingeniería Agronómica ubicada en la provincia del Azuay, Ecuador, Sur América. El objetivo fue caracterizar a los estudiantes matriculados en el periodo marzo - agosto 2016 y establecer los determinantes del rendimiento académico universitario, se aplicó al 100\% de la población estudiantil una encuesta estructurada sobre aspectos sociodemográficos y de satisfacción sobre aspectos educativos usando una escala de Likert y se analizó las bases de datos de los Sistemas de Gestión Socio Económica (SGSE) y de Gestión Académica (SGA) de la Universidad de Cuenca agrupando 48 posibles determinantes personales, sociales e institucionales. Para el análisis de datos se usó el paquete estadístico IBM® SPSS® Statistics versión 24 y el software Microsoft Office Excel® versión 16.0 considerando las herramientas apropiadas para una investigación descriptiva y correlacional. El estudio evidenció que la mayor población estudiantil proviene del área de influencia directa de la Universidad de Cuenca y de colegios fiscales con una valoración de rendimiento en el bachillerato de muy buena el mismo que no logran ser mantenido y/o superado por la mayoría de estudiantes en el transcurso de su formación de tercer nivel disminuyendo su rendimiento a una valoración de regular o buena. De los determinantes estudiados únicamente 18 muestran relación con el rendimiento académico universitario, aunque esta es mínima o baja.

Palabras clave: caracterización; universidad; correlación; determinantes sociales; determinantes personales; determinantes institucionales.

\section{Abstract}

The present descriptive correlational and transversal research was carried out in the Faculty of Agricultural Sciences of the University of Cuenca, Careers in Veterinary Medicine and Zootechnics and Agronomic Engineering located in the province of Azuay, Ecuador, South America. The objective was to characterize the students enrolled in the period March - August 2016 and establish the determinants of university academic performance, 100\% of the student population was applied a structured survey on sociodemographic aspects and satisfaction on educational aspects using a Likert scale and analyzed the databases of the Socio-Economic 
Management Systems (SGSE) and Academic Management (SGA) of the University of Cuenca, grouping 48 possible personal, social and institutional determinants. For data analysis, the statistical package IBM® SPSS® Statistics version 24 and Microsoft Office Excel® version 16.0 software were used, considering the appropriate tools for a descriptive and correlational investigation. The study showed that the largest student population comes from the area of direct influence of the University of Cuenca and tax colleges with a performance rating in the baccalaureate of very good the same that fail to be maintained and/or overcome by the majority of students in the course of their third level training, reducing their performance to a regular or good rating. Of the determinants studied, only 18 show a relationship with university academic performance, although this is minimal or low.

Keywords: characterization; university; correlation; social determinants; personal determinants; institutional determinants.

\section{Resumo}

A presente pesquisa descritiva correlacional e transversal foi realizada na Faculdade de Ciências Agrárias da Universidade de Cuenca, Carreiras em Medicina Veterinária e Zootecnia e Engenharia Agronômica localizada na província de Azuay, Equador, América do Sul. O objetivo foi caracterizar os alunos matriculados no período março-agosto de 2016 e estabelecer os determinantes do desempenho acadêmico da universidade, aplicado a $100 \%$ da população estudantil um inquérito estruturado sobre aspectos sócio-demográficos e satisfação com aspectos educacionais utilizando uma escala de Likert e analisou as bases de dados dos Sistemas de Gestão Socioeconômica (SGSE) e Gestão Acadêmica (SGA) da Universidade de Cuenca, agrupando 48 possíveis determinantes pessoais, sociais e institucionais. Para a análise dos dados, utilizou-se o pacote estatístico IBM® SPSS ${ }^{\circledR}$ Statistics versão 24 e o software Microsoft Office Excel@ versão 16.0, considerando as ferramentas apropriadas para uma investigação descritiva e correlacional. O estudo mostrou que a maior população de estudantes vem da área de influência da Universidade de Cuenca e escolas públicas com uma avaliação do desempenho na escola muito boa o mesmo que deixar de ser mantida e / ou ultrapassado pela maioria dos estudantes no decorrer de seu treinamento de terceiro nível, reduzindo seu desempenho a uma classificação 
regular ou boa. Dos determinantes estudados, apenas 18 mostram relação com o desempenho acadêmico universitário, embora seja mínimo ou baixo.

Palavras chave: caracterização; universidade; correlação; determinantes sociais; determinantes pessoais; determinantes institucionais.

\section{Introducción}

Con la emisión del Mandato 14 se establece la obligatoriedad de implementar procesos de evaluación en la universidad ecuatoriana los cuales fueron implementados por el Consejo de Evaluación, Acreditación y Aseguramiento de la Calidad de la Educación Superior (CEAACES) a través de un modelo genérico de autoevaluación de carreras que contempla cinco criterios: docencia, plan curricular, academia, ambiente institucional y estudiantes. Bajo dicha premisa todas las instituciones universitarias vieron la necesidad de implementar procesos de evaluación interna que les conduzca a mejorar la oferta académica sustentada en un diagnóstico situacional que les proyecte a procesos de mejora continua.

Es por ello, que en ese marco los estudiantes se convierten en los actores sujetos fundamentales dentro de la comunidad universitaria al ser los beneficiarios directos de la formación académica para su profesionalización, alrededor de estos giran aspectos sociales, económicos, familiares y de educación que de hecho repercuten sobre el rendimiento académico individual; el conocer su realidad facilita el entendimiento global a partir de lo cual se generan decisiones administrativas y académicas dentro la Institución de Educación Superior (IES) para alcanzar estándares de calidad altos (Muñoz, Sandoval, \& González, 2015), (Fernández, Álvarez, \& Colunga, 2006; Moscoloni, De Luján, Calvo, \& Isern, 2007).

Es así que Casillas, Chain, \& Jácome (2007) dicen:

Cuando en la educación superior se habla de los estudiantes, con frecuencia uno se topa con representaciones sociales de corte conservador que impiden ver y reconocer la realidad de los hechos: los estudiantes constituyen un grupo social diverso y no viven una experiencia escolar única. Padres, profesores, directivos, colegios profesionales y otros agentes desconocen lo que viven miles de jóvenes universitarios cotidianamente, ignoran lo que sienten los alumnos, apenas y reconocen la existencia de símbolos identitarios propios de esta clase de individuos; apenas 
tienen una ligera idea de lo que construyen intelectual y emocionalmente los jóvenes estudiantes de la educación superior. Las instituciones universitarias se están volviendo indiferentes ante los cambios que ocurren entre los estudiantes, porque simplemente no los conocen.

No cabe duda de que los estudiantes a través de su rendimiento académico contribuyen de manera sustantiva a la determinación de la calidad de las Instituciones de Educación Superior (Garbanzo, 2007), a pesar de que este es el resultado en el que confluyen múltiples factores de diversa complejidad y origen (Barahona \& Aliaga, 2013; Ocaña, 2011), que ha llevado a nivel mundial a proponer diversas metodologías para su valoración desde aquellas que contempla el éxito personal alcanzado hasta las más básicas y de uso común en la Universidad Ecuatoriana como es la acumulación de puntaje proveniente de evaluaciones escritas y diversas actividades calificadas sean estas de trabajo autónomo o de aula (Garbanzo, 2007; Villamizar \& Romero, 2011).

Durón y Oropeza (1999) citado por Izar, Ynzunza, \& López (2011) mencionan la existencia de cuatro factores que afectan el rendimiento académico (fisiológicos, pedagógicos, psicológicos y sociológicos) siendo estos de necesaria consideración para entender al estudiante universitario como sujeto rodeado de aspectos sociales, afectivos, ambientales, entre otros (Escorcia, Peralta, \& Vilora, 2013) lo que le hace único e individual (Casillas, Chain, \& Jácome, 2007). Garbanzo (2007), en cambio engloba en tres tipos de determinantes que fueron tomados como referente para el proceso de investigación, y son:

Determinantes personales: denominados psicológicos y comportamiento frente a los estudios.

Determinantes sociales: relacionados con el entorno en el que se desenvuelve el estudiante.

Determinantes institucionales: relacionados con aspectos pedagógicos, infraestructura entre otros.

Es entonces fundamental considerar que, para las instituciones universitarias a nivel mundial, el valorar su eficacia y calidad resulta de suma importancia el analizar el rendimiento de los estudiantes mucho más si se sabe lo multifactorial y la individualidad originada por las diferencias del entorno social. A través de diversos estudios a nivel mundial se puede observar que no existe una definición única de factores que influyen sobre el rendimiento de los estudiantes universitarios, se mencionan como relevantes el medio sociocultural en que vive, nivel educativo, expectativas de padres a hijos, integración y participación social, carrera escogida y nota de bachillerato, el sexo, edad, nivel educativo de los padres y estado laboral de 
los estudiantes, Artunduaga (2008), Barahona \& Aliaga (2013); Di Grecia (2007) y Armenta, Pacheco, \& Pineda (2008).

No es menos cierto que la motivación tiene influencia sobre el rendimiento académico del estudiante, factor enmarcado en los determinantes psicológicos concebida como el estado interno de la persona que provoca una conducta o como una fuerza o conjunto de fuerzas que provoca acciones para satisfacer necesidades y cumplir metas personales o sociales (Enriquez, Lemus, \& Reyes, 2012), reconociéndose dos tipos de motivación: la motivación extrínseca causada factores externos positivos o negativos y la motivación intrínseca relacionada con la satisfacción en base a la instrumentación de acciones propias. Si bien este factor cognitivo-emocional tienen alta subjetividad es importante investigarlo desde la caracterización ya que denota la voluntad personal del estudiante para emprender procesos de enseñanza aprendizaje y el desempeño general del estudiante universitario frente a sus obligaciones estudiantiles. (Núñez, 2009; Maquillón \& Hernández, 2011).

\section{Materiales y Métodos}

La investigación se realizó en la Facultad de Ciencias Agropecuaria de la Universidad de Cuenca, Ecuador que cuenta con las Carreras de Medicina Veterinaria y Zootecnia e Ingeniería Agronómica, siendo de tipo descriptiva correlacional y con un carácter transversal, los sujetos investigados fueron el $100 \%$ de la población estudiantil del periodo académico marzo - agosto 2016. Se utilizó una encuesta estructurada y validada para la recolección de información primaria y como segunda fuente se analizó la base de datos oficial de los Sistemas de Gestión Socio Económica (SGSE) y de Gestión Académica (SGA) de la Universidad de Cuenca que contiene información sociodemográfica, económica y educativa de los estudiantes matriculados. En total se estudiaron 48 posibles determinantes del rendimiento académico agrupadas en personales, sociales e institucionales (Tabla 1). 
Tabla 1. Determinantes personales, sociales e institucionales

\begin{tabular}{|c|c|c|}
\hline Personales & Sociales & Institucionales \\
\hline $\begin{array}{l}\text { - Edad } \\
\text { - Estado civil } \\
\text { - Sexo } \\
\text { - Procedencia } \\
\text { - Nota de bachillerato } \\
\text { - Priorización de carrera } \\
\text { de acuerdo Sistema } \\
\text { Nacional de Nivelación } \\
\text { - } \text { Admisión } \\
\text { - Plan de vida } \\
\text { - Horas de estudio fuera } \\
\text { - clase } \\
\text { - Planificación de } \\
\text { - estudios } \\
\text { - Hábito de leer } \\
\text { - Alimentación diaria } \\
\text { - Mdecuada } \\
\text { - Motivación intrínseca }\end{array}$ & $\begin{array}{l}\text { - Tipo de ingreso a la } \\
\text { universidad } \\
\text { - Ciclo actual de estudio } \\
\text { - Tipo colegio de } \\
\text { procedencia } \\
\text { - Tipo de bachillerato } \\
\text { - Procedencia de ayuda } \\
\text { económica para estudio } \\
\text { - Motivación extrínseca } \\
\text { - Trabajo } \\
\text { - Horas por semana de } \\
\text { - } \text { trabajo } \\
\text { - Aargas familiares } \\
\text { - Acceso a internet en } \\
\text { domicilio } \\
\text { - Horas dedicadas a } \\
\text { redes sociales } \\
\text { - Práctica deportiva } \\
\text { - Lugar adecuado para } \\
\text { estudio en domicilio } \\
\text { - Participación activa } \\
\text { - Consumo de alcohol y } \\
\text { - tabaco } \\
\text { - } \text { consumo de drogas } \\
\text { - Nivetas a control educativo del } \\
\text { - Nadre } \\
\text { - Nivel educativo de la } \\
\text { madre } \\
\text { - Ocupación del padre } \\
\text { - Jefupación de la madre } \\
\text { - Sueldo de familia } \\
\text { - Número de integrantes } \\
\text { de familia }\end{array}$ & $\begin{array}{l}\text { Nivel de satisfacción con: } \\
\text { - Formación recibida } \\
\text { - Horario actual } \\
\text { - Guía y motivación } \\
\text { docente } \\
\text { - Uso de formas } \\
\text { alternativas en el } \\
\text { proceso de enseñanza } \\
\text { aprendizaje } \\
\text { - Claridad y concreción } \\
\text { docente } \\
\text { - Preparación de } \\
\text { docentes Forma de } \\
\text { evaluación } \\
\text { - Tipo de evaluación } \\
\text { aplicada } \\
\text { - Evaluación continua } \\
\text { - Uso de varias } \\
\text { actividades para } \\
\text { calificación de } \\
\text { aprovechamiento } \\
\text { - Extensión y claridad de } \\
\text { las evaluaciones } \\
\text { escritas } \\
\text { - Aulas adecuadas } \\
\text { laboratorios y granjas } \\
\text { funcionales } \\
\text { - Relación y } \\
\text { comunicación } \\
\text { respetuosa abierta y de } \\
\text { confianza con docentes }\end{array}$ \\
\hline
\end{tabular}


Para la valoración del rendimiento académico universitario real, se tomó en cuenta el promedio del puntaje alcanzado en las materias aprobadas y no aprobadas ya que este permite una valoración más objetiva del desempeño general del estudiante en su vida universitaria al considerar lo extendido que puede ser su proceso de titulación.

La tabulación y análisis se realizó con el uso del paquete estadístico IBM $®$ SPSS $®$ Statistics versión 24 y el software Microsoft Office Excel® versión 16.0. estableciendo gráficos descriptivos y posterior determinación del nivel de correlación y su significancia mediante la prueba de Spearman.

\section{Resultados}

La población de la Facultad de Ciencias Agropecuarias de la Universidad de Cuenca, a la fecha de la investigación contabilizó 813 estudiantes, 57\% matriculados en la Carrera de Medicina Veterinaria y Zootecnia (MVZ) de este 53\% de género femenino y 47\% masculino; el $43 \%$ pertenecen a la Carrera de Ingeniería Agronómica (IAG) compuesta de una mayoría de género masculino $55 \%$ y $45 \%$ femenino; la procedencia mayoritaria (gráfico 1) de la población estudiantil es la provincia de Azuay (MVZ 86,1\% e IAG 89,4\%) sede de la Universidad de Cuenca, seguido de Cañar (MVZ 9,54\% e IAG 7,7\%) y los porcentajes restantes corresponden a otras provincias.

MEDICINA VETERINARIA Y ZOOTECNIA

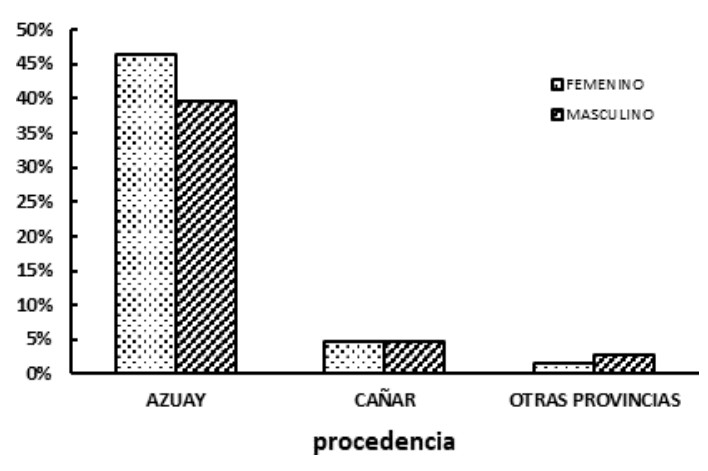

INGENIERÍA AGRONÓMICA

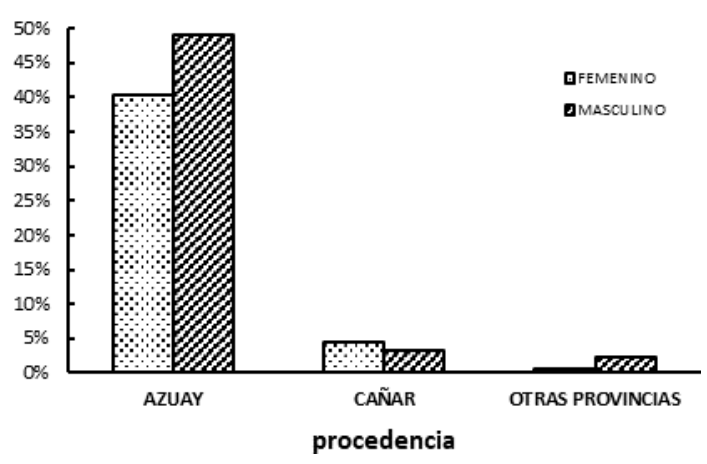

Gráfico 1. Población estudiantil de acuerdo a lugar de procedencia 
La educación fiscal es la más común en el Ecuador y genera el mayor número de bachilleres de la República. En las carreras de MVZ e IAG se refleja esa realidad, el 65,5\% y 79,2\% respectivamente proceden de colegios fiscales (gráfico 2), seguido de aquellos bachilleres de colegios particulares con un 20,2\% y 12,5\% respectivamente sobresaliendo en ambos casos el bachillerato en Ciencias Generales, los restantes porcentajes corresponden a colegios fiscomisionales o universitarios; se puede observar que el rendimiento académico universitario se comporta similar en todos los tipos de colegio de procedencia siendo mejor en la carrera de Ingeniería Agronómica al contar con un mayor porcentaje de estudiantes con una equivalencia de bueno en comparación con el mayoritario regular de la carrera de Medicina Veterinaria y Zootecnia.
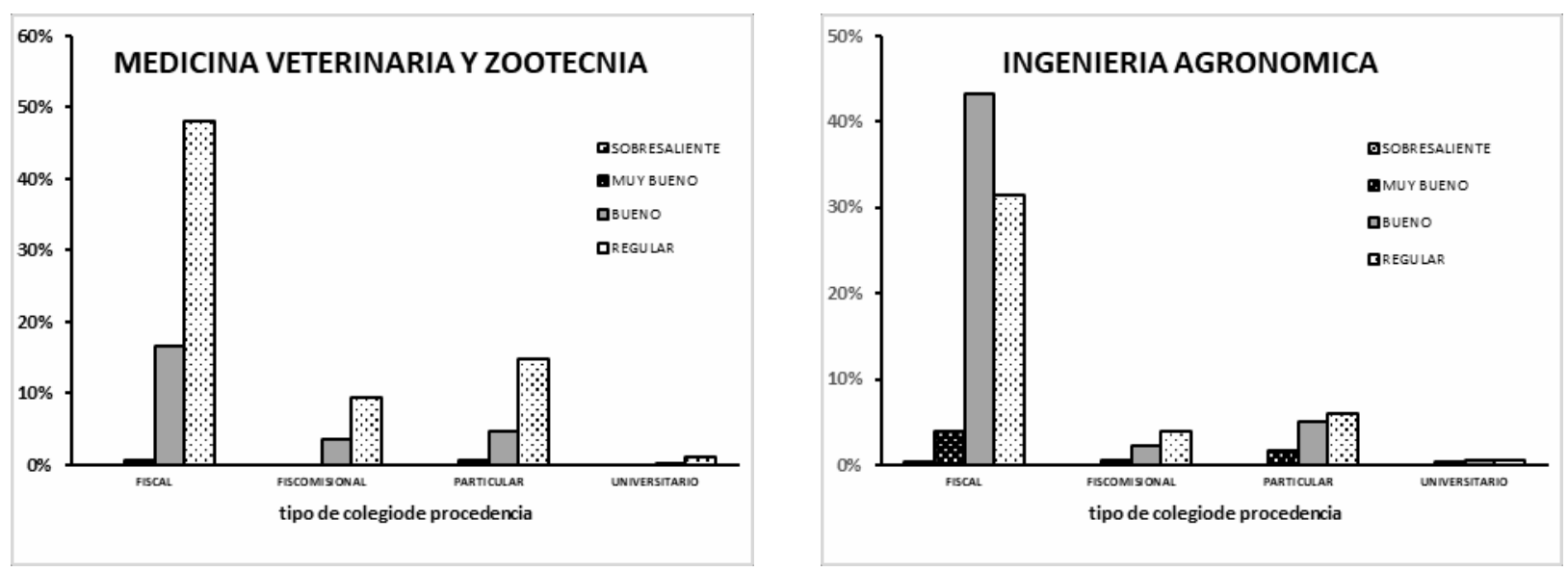

Gráfico 2. Población estudiantil por tipo de colegio de procedencia y rendimiento académico universitario.

Muchas veces se discute sobre el nivel de preparación con el que llegan los bachilleres a la educación superior con el prejuicio de la existencia de mejor preparación en la educación particular que en la pública considerándose la posibilidad de un mejor rendimiento académico. Si observamos el comportamiento de la población estudiada se puede notar que el mayor porcentaje de estudiantes ingresaron a la universidad con un rendimiento en el bachillerato de 8.1 - 9 que corresponde a una equivalencia de muy buena existiendo similitud de población en las dos carreras tanto en su tipo de colegio de origen como en su nota de bachillerato (gráfico 3). 


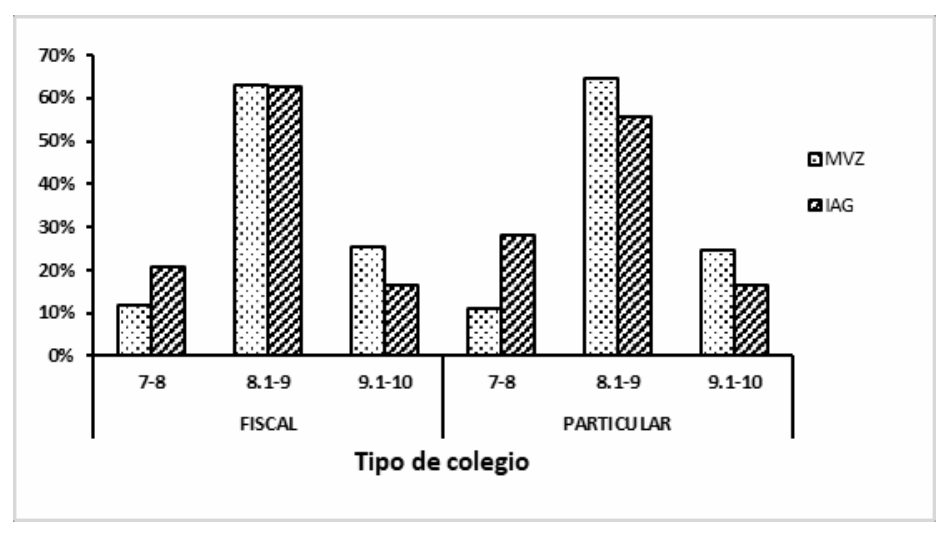

Gráfico 3. Población estudiantil total por tipo de colegio de procedencia y nota de bachillerato.

Resulta preocupante que los estudiantes de las Carreras estudiadas no logran mantener en la universidad el rendimiento académico alcanzado en el bachillerato siendo generalizada la disminución en los estudios superiores pasando de una equivalencia de muy buena a una equivalencia de regular (6 a 7 sobre 10) en Medicina Veterinaria y Zootecnia y de buena (7 a 8 sobre 10) en Ingeniería Agronómica (gráfico 4), sin mostrar diferencias al considerar la especialidad conseguida en el bachillerato (gráficos 5) lo que indicaría un nivel similar de conocimientos previos con el que llegan los estudiantes a la educación superior.
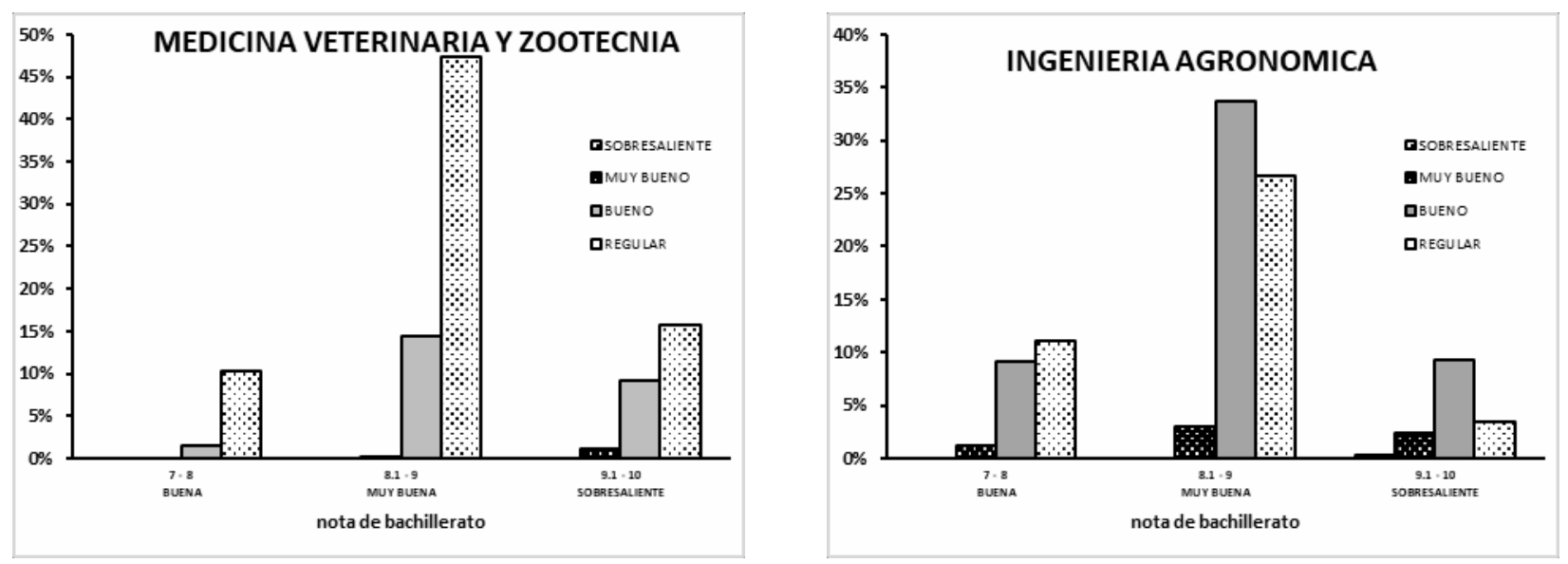

Gráfico 4. Población estudiantil de acuerdo a nota de bachillerato y rendimiento académico universitario. 

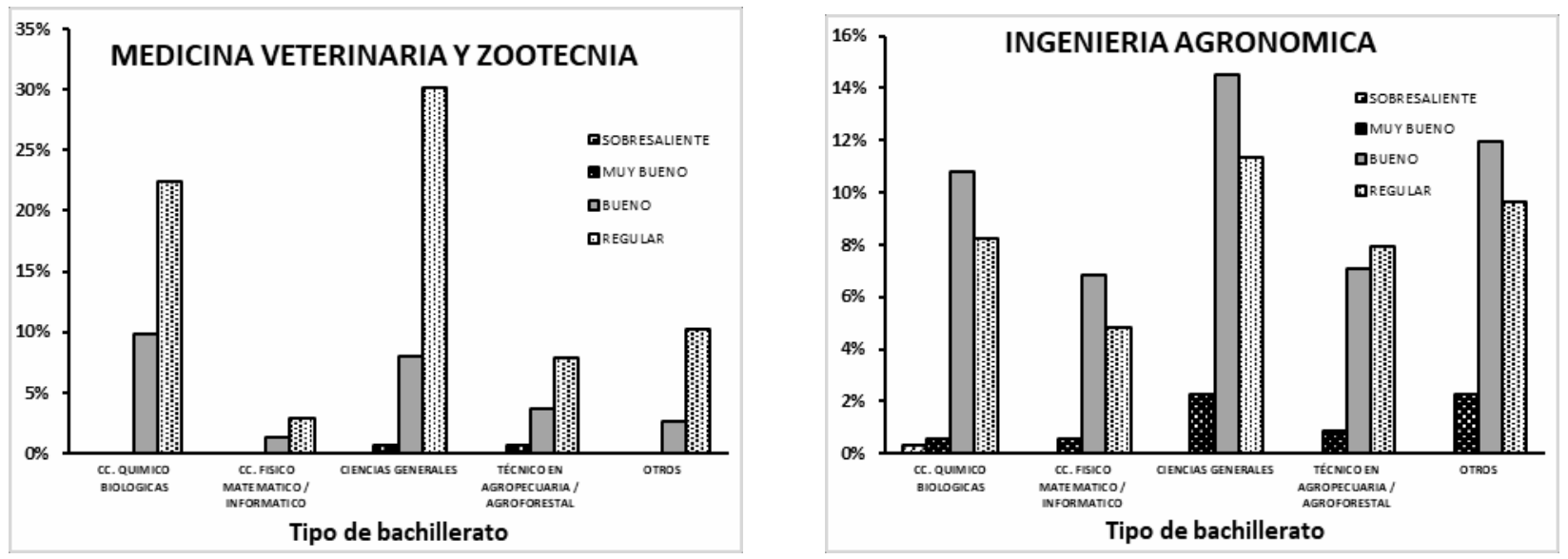

Gráfico 5. Población estudiantil por carrera, tipo de bachillerato y rendimiento académico universitario

Lo descrito sin lugar a dudas debería constituir una constante preocupación de la IES mucho más si el 70,4\% y 77,6\% de la población estudiantil de las Carreras de MVZ e IAG ingresó a través de la aprobación del curso de nivelación mediante la evaluación de conocimientos y capacidades y la obtención de una nota mínima de acuerdo a la carrera escogida y un 14,9 \% y 11,8\% mediante el ingreso directo por aprobación de un examen de exoneración al proceso de nivelación (gráfico 6); claro está que con seguridad coexiste responsabilidad sobre el rendimiento académico de los estudiantes por tener el carácter de multifactorial al encontrarse involucrados factores individuales del estudiante, el nivel y calidad de formación que el estudiante trae desde el bachillerato como conocimientos previos y la formación tanto en metodología y calidad recibida en la educación superior.
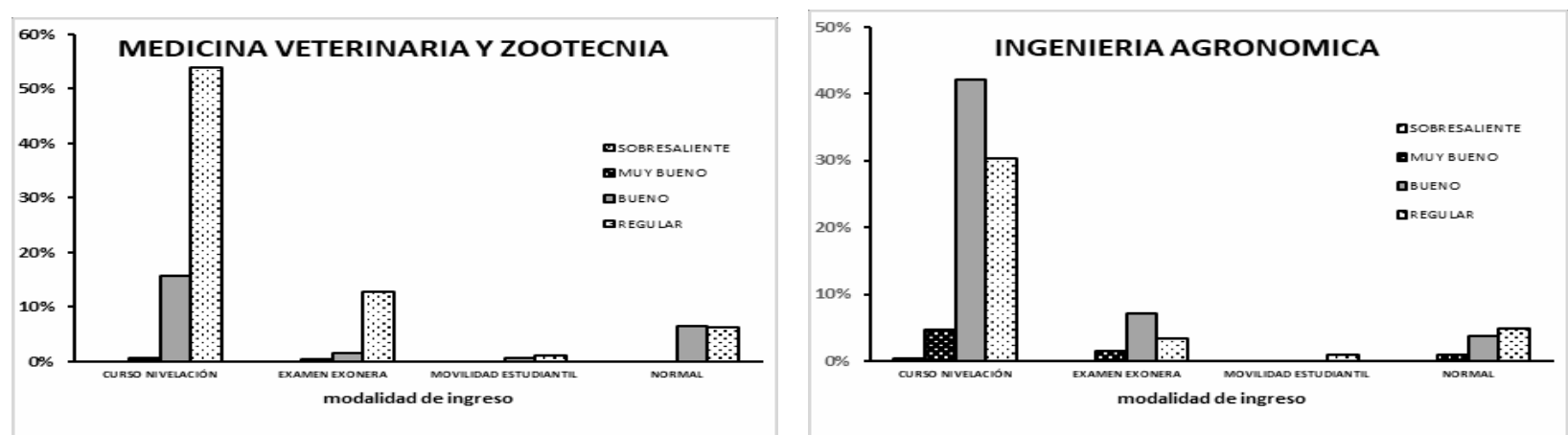

Gráfico 6. Población estudiantil según rendimiento académico y modalidad de ingreso. 
Analizadas las determinantes de diferente tipo, 18 de ellas muestran relación significativa con el rendimiento académico universitario de los estudiantes 11 ( $r<0,001)$ y 7 ( $r<0,005)$, sin embargo, dicha correlación es mínima o baja (Tabla 2). De todas ellas, la variable carrera es la que presenta el mayor grado de relación pudiendo deducirse que los estudiantes de las Carreras investigadas muestran diferencias en su rendimiento académico.

Tabla 2. Niveles de correlación de rendimiento académico con diferentes variables.

\begin{tabular}{|c|c|c|c|}
\hline Variable & $\begin{array}{c}\text { Coeficiente } \\
\text { de } \\
\text { correlación }\end{array}$ & $\begin{array}{c}\text { Sig. } \\
\text { (bilateral) }\end{array}$ & $\mathbf{N}$ \\
\hline \multicolumn{4}{|l|}{ Determinantes personales } \\
\hline Carrera & $0,323 * *$ & 0,000 & 813 \\
\hline Forma de ingreso & $0,171 * *$ & 0,000 & 416 \\
\hline Nota de bachillerato & $0,127 * *$ & 0,000 & 787 \\
\hline Opción de carrera elegido de acuerdo Sistema & $-0,120 * *$ & 0,002 & 665 \\
\hline Plan de vida & $-0,083 *$ & 0,019 & 801 \\
\hline Planifica sus estudios & $0,126 * *$ & 0,000 & 812 \\
\hline \multicolumn{4}{|l|}{ Determinantes sociales } \\
\hline Origen de ayuda económica & $0,117 * *$ & 0,001 & 764 \\
\hline Trabaja & $0,105 * *$ & 0,003 & 804 \\
\hline Cargas familiares & $0,074 *$ & 0,036 & 802 \\
\hline Cuenta con alimentación adecuada & $0,090 *$ & 0,010 & 813 \\
\hline Jefatura de familia & $0,082 *$ & 0,021 & 794 \\
\hline \multicolumn{4}{|l|}{ Determinantes institucionales } \\
\hline Satisfacción con horario de clases & $-0,170 * *$ & 0,000 & 811 \\
\hline Obtención de guía y motivación en aula & $0,072 *$ & 0,040 & 811 \\
\hline $\begin{array}{l}\text { Uso de múltiples actividades para } \\
\text { aprovechamiento }\end{array}$ & $0,081 *$ & 0,021 & 808 \\
\hline $\begin{array}{l}\text { Claridad y extensión adecuada de evaluaciones } \\
\text { escritas }\end{array}$ & $0,070 *$ & 0,045 & 811 \\
\hline Aulas adecuadas para aprendizaje & $-0,121 * *$ & 0,001 & 810 \\
\hline $\begin{array}{l}\text { Dotación adecuada de laboratorios y granjas } \\
\text { para prácticas }\end{array}$ & $-0,154 * *$ & 0,000 & 807 \\
\hline $\begin{array}{l}\text { Relación y comunicación respetuosa abierta y } \\
\text { de confianza }\end{array}$ & $0,107 * *$ & 0,002 & 813 \\
\hline
\end{tabular}

** la correlación es significativa en el nivel 0,01

* la correlación es significativa en el nivel 0,05 
Variables como motivación extrínseca e intrínseca, acceso a servicio de internet, lugar adecuado para estudio en domicilio, frecuencia de lectura, práctica deportiva autónoma, consumo de alcohol, tabaco y drogas sujetas a control, el nivel educativo y ocupación del padre y la madre no muestran relación con el rendimiento académico.

\section{Conclusiones}

Los estudiantes de la Facultad de Ciencias Agropecuarias de la Universidad de Cuenca al periodo marzo-agosto 2016 se muestra equilibrada en cuanto a género, en su mayoría provienen de la provincia del Azuay por lo que se puede decir que el centro de educación superior cumple con el propósito de servir a la región en donde se halla asentada.

La mayoría de estudiantes provienen de la educación fiscal con un bachillerato en ciencias generales con un rendimiento equivalente a muy buena, no se observan diferencias en su rendimiento universitario de acuerdo a las variables mencionadas.

Los estudiantes que cursan las carreras de la Facultad de Ciencias Agropecuarias no logran sostener el rendimiento académico conseguido en el bachillerato, notándose una clara y preocupante disminución del mismo en la Educación Superior.

No se encuentra una relación fuerte entre las variables estudiadas y el rendimiento académico universitario alcanzado por los estudiantes de la Facultad de Ciencias Agropecuarias determinándose correlaciones mínimas y bajas; esto muy probablemente se deba al carácter multifactorial que tiene el rendimiento académico.

\section{Referencias Bibliográficas}

Armenta, N., Pacheco, C., \& Pineda, E. (2008). En Factores Socioeconómicos que intervienen en el desempeño académico de los estudiantes universitarios de la Facultad de Ciencias Humanas de la Universidad Autónoma de Baja California. Revista IIPSI Facultad de Psicología UNMSM, $153-165$. 
Artunduaga, M. (2008). Variables que influyen en el rendimiento académico en la Universidad. (Tesis Doctoral), Universidad Complutense de Madrid, Departamento MIDE (Métodos de Investigación y Diagnóstico en Educación), Madrid.

Barahona, P., \& Aliaga, V. (2013). Variables predictoras del rendimiento académico de los alumnos de primer año de las carreras de Humanidades de la Universidad de Atacama, Chile. (D. d. Asunción, Ed.) Revista Internacional de Investigación en Ciencias Sociales, 9(2), 207-220.

Casillas, M., Chain, R., \& Jácome, N. (2007). Origen Social de los Estudiantes y Trayectorias Estudiantiles en la Universidad Veracruzana. Revista de la Educación Superior, XXXVI (2) (142), 7 - 29. Recuperado el 30 de Agosto de 2017

Di Grecia, L. (2007). En el Rendimiento Académico Universitario. La Plata, Argentina. Recuperado el 31 de julio de 2017

Enriquez, V., Lemus, C., \& Reyes, E. (2012). Caracterización de los estudiantes de Pedagogía en Educación Media, Mención Educación Física, de la Universidad de los Lagos, Puerto Montt. Revista Horizonte, 3(1), 17-24. Recuperado el 26 de Julio de 2017, de http://revistahorizonte.ulagos.cl/index.php/Rev_Horizonte/article/view/33

Escorcia, R., Peralta, A., \& Vilora, J. (2013). Caracterización de los estudiantes de la Universidad Popular del César de acuerdo a las variables socioeconómicas, psicosociales y geográficas. Universidad Popular del César, Valledupar. Recuperado el 30 de julio de 2017

Fernández, I., Álvarez, N., \& Colunga, S. (2006). Caracterización psicopedagógica. (EcuRed, Ed.) Obtenido de Centro de Estudios de Ciencias de la Educación Superior "Enrique José Varona" Universidad de Camaguey https://www.ecured.cu/Caracterizaci\%C3\%B3n_psicopedag\%C3\%B3gica

Garbanzo, G. (2007). Factores asociados al rendimiento académico en estudiantes universitarios, una reflexión desde la calidad de la educación superior pública. Revista Educación, 1(31), 43-63. Recuperado el 15 de mayo de 2016 
Izar, J., Ynzunza, C., \& López, H. (2011). Factores que afectan el desempeño académico de los estudiantes de nivel superior en Rioverde, San Luis Potosí, México. (I. d.-U. Veracruzana, Ed.) Revista de Investigación Educativa (12), 1-18. Recuperado el 12 de mayo de 2016, de http://www.redalyc.org/articulo.oa?Id=283121721005

Maquillón, J., \& Hernández, F. (2011). Influencia de la motivación en el rendimiento académico de los estudiantes de formación profesional. (A. U. Profesorado, Ed.) Revista Electrónica Interuniversitaria de Formación del Profesorado, 14(1), 81- 100. Recuperado el 30 de julio de 2017, de http://www.redalyc.org/articulo.oa?Id=217017192007

Moscoloni, N., De Luján, M., Calvo, S., \& Isern, G. (2007). Comparación de perfiles sociales de estudiantes universitarios a través de técnicas de visualización de objetos simbólico. Revista Iberoamericana de Educación, 1-9.

Muñoz, E., Sandoval, M., \& González, L. (2015). Caracterización de los estudiantes de primer año de Medicina y Tecnología Médica, Universidad de San Sebastián, sede Concepción, en base a variables asociadas al entorno familiar. TS Cuadernos de Trabajo Social, 11, 1-17.

Núñez, J. (2009). Motivación, aprendizaje y rendimiento académico. En U. d. Minho (Ed.), Actas do X Congresso Internacional Galego-Português de Pedagogía, (págs. 41 - 67). Braga. Recuperado el 30 de julio de 2017

Ocaña, Y. (2011). Variables académicas que influyen en el rendimiento académico de los estudiantes universitarios. Revista Investigación educativa, 165-179.

Villamizar, G., \& Romero, L. (2011). Relación entre variables psicosociales y rendimiento académico en estudiantes de primer semestre de Psicología. Revista Educación y Desarrollo Social, 5(1), 41-54. Recuperado el 15 de mayo de 2016. 